\title{
Design And Simulation Noise Characteristics of AlGaN/GaN/AIGaN/GaN HEMT on SIC Substrate For Low Noise Applications
}

\author{
Yasaman Najmabadi ${ }^{1}$, Alireza Kashaniniya ${ }^{1}$ \\ ${ }^{\text {I}(D e p a r t m e n t ~ o f ~ E l e c t r i c a l ~ E n g i n e e r i n g, ~ C e n t r a l ~ T e h r a n ~ B r a n c h, ~ I s l a m i c ~ A z a d ~ U n i v e r s i t y, ~ T e h r a n, ~ I r a n) ~}$
}

\begin{abstract}
In this paper, AlGaN/GaN/AlGaN/GaN high electron mobility transistor (HEMT) with 0.25 um gatelength have been designed on an SIC-4H substrate. DC and Noise characteristics of AlGaN/GaN/AlGaN/GaN HEMT with 0.25 um gate-length at microwave frequencies have been explored. The simulation has been performed by using the Silvaco software. The extrinsic transconductance of the device was $350 \mathrm{~ms} / \mathrm{mm}$. Also, device exhibited current drive capability as high as $1750 \mathrm{ma} / \mathrm{mm}$. The device has demonstrated high unity current gain cut-off frequency (ft) of $200 \mathrm{GHz}$. The microwave noise characteristics of the device were determined from 0 to $20 \mathrm{GHz}$ at different drain biases and drain currents. At a gate bias of $-3 \mathrm{~V}$ and drain bias of $10 \mathrm{~V}$, device exhibited a minimum noise figure (NFmin) of $0.35 \mathrm{~dB}$ and maximum associated gain (Gma) of $24.35 \mathrm{~dB}$ at $10 \mathrm{GHz}$. The noise resistance of device is $7.2 \mathrm{ohm}$ at $10 \mathrm{GHz}$, which is very suitable for low noise applications in X-band frequency range. These results indicates the capability of AlGaN/GaN HEMT for low noise and high power amplifiers.
\end{abstract}

Keywords: AlGaN, GaN, HEMT, microwave noise, minimum noise figure

\section{Introduction}

For microwave high-power and low noise applications AlGaN/GaN HEMT attracted consideration because of their excellent microwave characteristics, low noise and high power microwave performance and high current drive capability [1]-[2].The main target of novel electronics technology is the fabrication of faster devices with the highest level of power and lowest level of noise figure [3]. Recently, heterostructure devices have provided superior performance than MESFETS. Preliminary researches have shown that noise properties of AlGaN/GaN-HEMTs are comparable to those of AlGaAs/GaAs HEMTs [2]. AlGaN/GaN-HEMT with 0.25 um gate-lengh has demonstrated NFmin of $0.77 \mathrm{~dB}$ at $5 \mathrm{GHz}$ and NFmin of $1.06 \mathrm{~dB}$ at $10 \mathrm{GHz}$ [4]. $\mathrm{AlGaN} / \mathrm{GaN}-\mathrm{HEMT}$ on $\mathrm{SiC}$ with a gate length of $0.15 \mathrm{um}$ showed NFmin of $0.6 \mathrm{~dB}$ at $10 \mathrm{GHz}$ [5]. An NFmin of $0.98 \mathrm{~dB}$ at $18 \mathrm{GHz}$ was achived in AlGaN/GaN-HEMT with a gate-length of 0.12 um [1]. $0.2 \mathrm{um}$ $\mathrm{AlGaN} / \mathrm{GaN}-\mathrm{HEMT}$ with a NFmin of $1.5 \mathrm{~dB}$ at $26 \mathrm{GHz}$ was reported [6]. AlGaN/GaN-HEMT with $0.25 \mathrm{um}$ gate-length has demonstrated NFmin of $0.75 \mathrm{~dB}$ at $10 \mathrm{GHz}$ [3].The purpose of this paper is to design of $A l_{0.3} G a_{0.7} \mathrm{~N} / \mathrm{GaN} / \mathrm{Al}_{0.06} G a_{0.94} \mathrm{~N} / \mathrm{GaN}$ HEMT transistor with extremely low noise figure while handling sufficient high power. The 2-D device simulator Silvaco ATLAS software is used for designing and simulation of device. The Silvaco software has high strong capability in semiconductor design and simulation analysis. Device exhibited NFmin of $0.35 \mathrm{~dB}$ and maximum associated gain of $24.35 \mathrm{~dB}$ at $10 \mathrm{GHz}$. Also, device has demonstrated NFmin of $0.51 \mathrm{~dB}$ and maximum associated gain of $22.3 \mathrm{~dB}$ at $18 \mathrm{GHz}$. To our knowledge, these are the best microwave noise characteristics of any GaN HEMTs ever reported with a 0.25 um gate-length. The main task of this paper is explained completely in the following manner. The first part of this paper explains the device structure and layers detail. In the second part of this paper simulation results are explained. In this section, DC characteristics and microwave noise performance of device is explored. In the final part, the complete conclusion of this paper is explained.

\section{Device Structures}

The cross section of the AlGaN/GaN/AlGaN/GaN HEMT with 0.25 um-gate-length is shown in fig. 1 . The layers are grown on a 3-um SIC-4H substrate. The structure consists of a $2.5-\mu \mathrm{m}$ - thick undoped GaN buffer layer, a 21- nm-thick AlGaN bottom barrier layer with the $\mathrm{Al}$ composition 6\%, a 14-nm-thick GaN channel layers and a 24 -nm-thick $\mathrm{Al} 0.3 \mathrm{Ga} 0.7 \mathrm{~N}$ top barrier layer. In order to optimize the channel electron density and comfort good ohmic contacts the AlGaN schottky layer is selectively doped with Si [2]. Gate-source and gate-drain spacing is equal to $0.5 \mathrm{um}, 0.75 \mathrm{um}$ respectively. The metal work function for the gate schottky contact is $(4.9 \mathrm{ev})$. The material parameters of $\mathrm{AlGaN}$ and $\mathrm{GaN}$ semiconductor are also included into the simulation that summarized in table I [7]. 


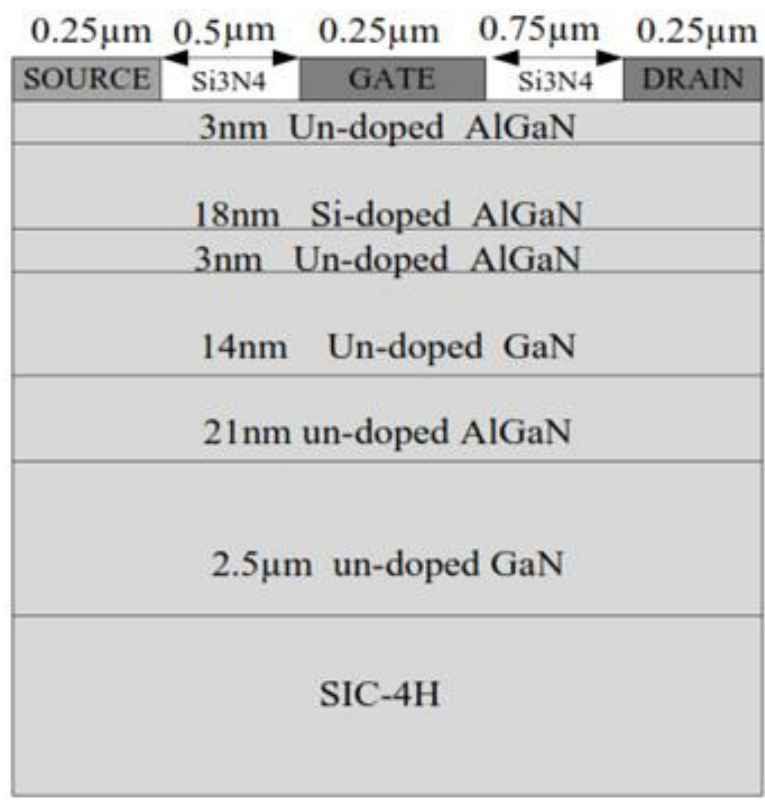

Fig. 1. Cross section of the AlGaN/GaN/AlGaN/GaN HEMT with 0.25 um-gate-length

Table I. the material parameters of $\mathrm{AlGaN}$ and $\mathrm{GaN}$ semiconductor

\begin{tabular}{|l|l|l|l|}
\hline Material & Mobility $(\mu)$ & Band gap (Eg) & Affinity \\
\hline$A l_{0.3} G a_{0.7} N$ & 870 & $4.023(\mathrm{ev})$ & 2.97 \\
\hline$A l_{0.06} G a_{0.94} N$ & 950 & 3.55 & 3.35 \\
\hline$G a N$ & 1300 & $3.42(\mathrm{ev})$ & 3.42 \\
\hline
\end{tabular}

The polarization bar exists in the HEMT transistor based on GaN, as a result the high drive current density is obtained. The polarization bar phenomena has been adapted in our simulation. In order to take the spontaneous and piezoelectric polarization into account in regions, POLARIZATION and CALC.STRAIN model is included. K.P model has been considered in our simulation for calculation effective masses and band gap energies in drift-diffusion device. To take account of recombination effects, we recommend the use of the Shockley-Read-Hall (SRH) model. High electric filed velocity saturation is modelled through the filed related mobility model (FLDMOB). Concentration mobility (CONMOB) model has been considered for estimation of the concentration dependent mobility effect. Auger model is used for recombination accounting for high level injection effects. Fig. 2 shows the conduction band profile in an AlGaN/GaN HEMT at the equilibrium state. The two minimum point in the conduction band profile, placed at $.029 \mathrm{um}$ and $0.059 \mathrm{um}$, are clearly seen.

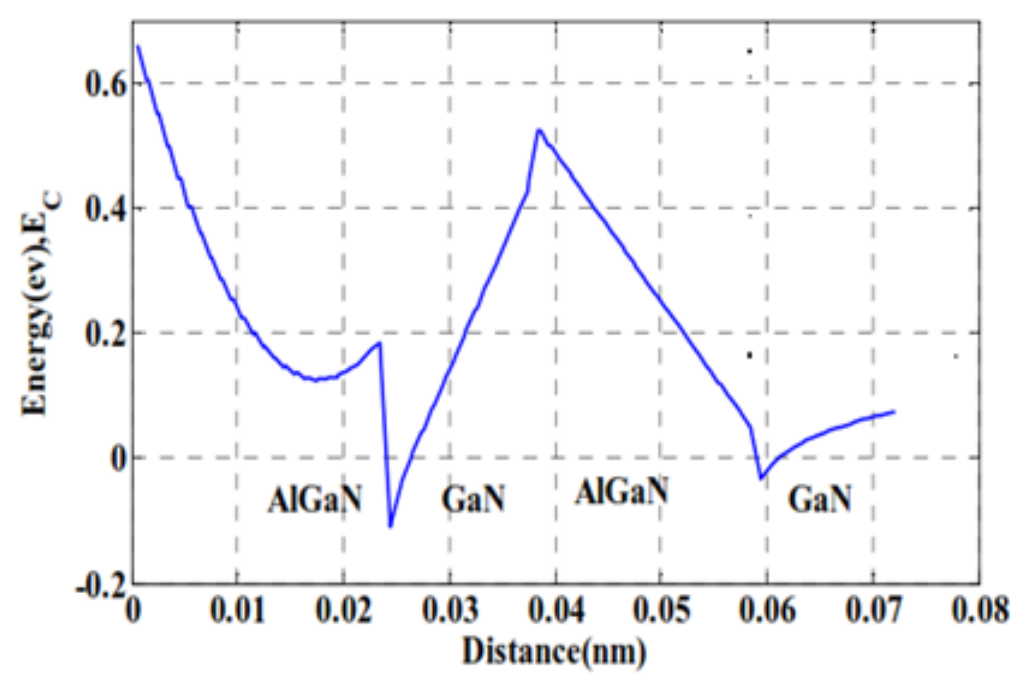

Fig. 2. Conduction band diagram $\mathrm{Ec}$ of $\mathrm{AlGaN} / \mathrm{GaN} / \mathrm{AlGaN} / \mathrm{GaN}-\mathrm{HEMT}$ at the equilibrium state 


\section{Dc Characteristic}

Current-voltage (I-V) characteristics of a AlGaN/GaN HEMT is simulated for the gate biases (Vgs) from $0 \mathrm{~V}$ to $-6 \mathrm{~V}$ in step of $0 \mathrm{~V}$, and for the drain biases from 0 to $15 \mathrm{~V}$, which is indicated in Fig. 3. Device depicted very high drain current drive capability. The maximum drain current was $1750 \mathrm{ma} / \mathrm{mm}$ at a gate bias of $0 \mathrm{~V}$ and a drain bias of a $13.5 \mathrm{~V}$.

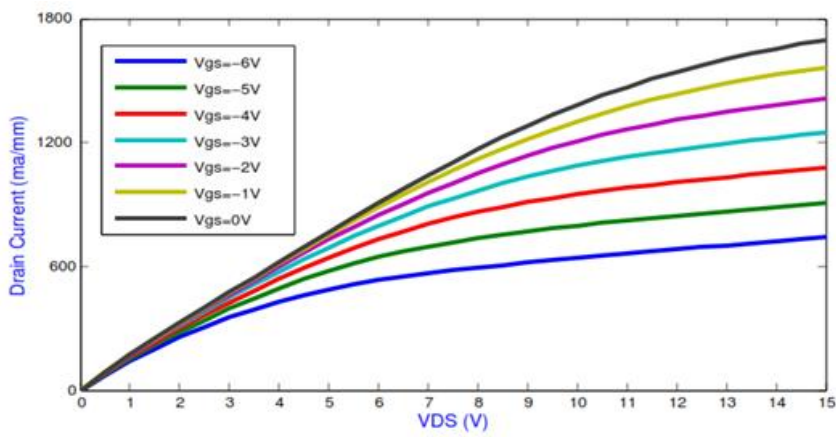

Fig. 3. I-V characteristics of $\mathrm{AlGaN} / \mathrm{GaN} / \mathrm{AlGaN} / \mathrm{GaN}$ HEMT , the gate bias was swept from $0 \mathrm{~V}$ to $-6 \mathrm{~V}$

The dc transfer characteristics (ID-VGS) and transconductance of the $0.25 \mathrm{um} \mathrm{AlGaN/GaN} \mathrm{HEMT} \mathrm{are}$ shown in Fig. 4 and Fig. 5. In both figures, the gate-source voltage (Vgs) varies between -15 to 0 volt with a step $0.5 \mathrm{~V}$. According to fig. 5, gate voltage increment results in an increased transconductance, because of the fact that the increased gate voltage causes the carrier concentration increasing in the channel. By more increasing the gate voltage, maximum transconductance is obtained. After this point, the transconductance will be decreased. In fact, a large gate voltage decreases the gate control on the channel, hence transconductance is reduced. By more increasing the gate voltage, vertical electric filed is increased in the channel, this filed causes the electrons mobility decrease in the channel, and hence, transconductance will be reduced. Drain voltage increment results in an increase in the gate control on the channel. Then transconductance is increased. Because of the increased drain voltage causes the electron concentration reduce in the parasitic channel in AlGaN layer. By more increasing the drain voltage, lateral electric filed and electrons velocity will be increased in the channel and maximum transconductance is obtained. A peak extrinsic transconductance of $350 \mathrm{~ms} / \mathrm{mm}$ was achieved at $\mathrm{Vgs}=-5 \mathrm{~V}$ and $\mathrm{Vds}=12 \mathrm{~V}$.

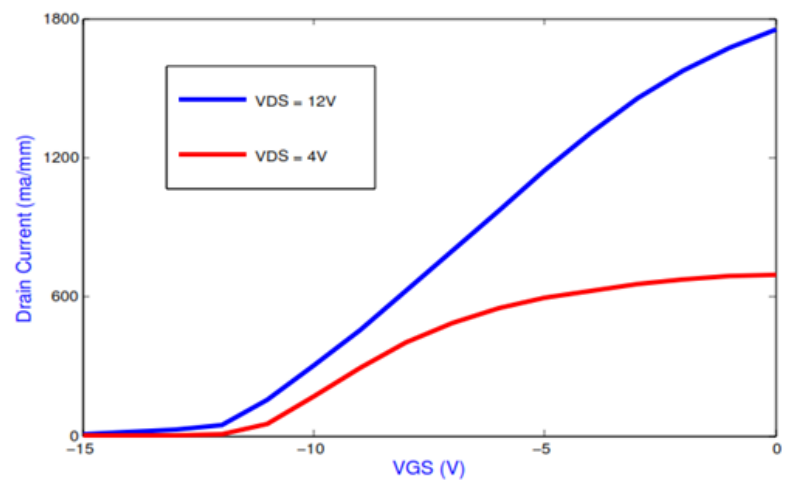

Fig. 4. (Id-Vgs) for $\mathrm{AlGaN} / \mathrm{GaN}$ HEMT

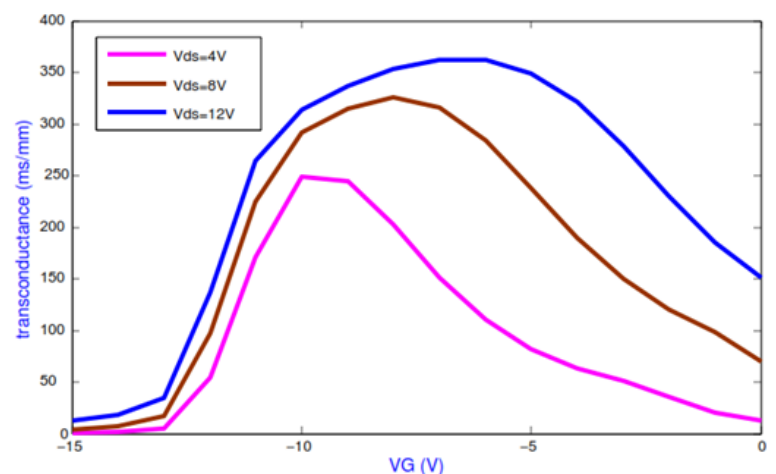

Fig. 5. (transconductance-VG) at the different drain-source biases 


\section{Microwave Noise Performance}

Noise is very important factor in AlGaN/GaN HEMT transistor. Impact ionization, tunneling current, change of carrier velocity, carrier generation and recombination provide the noise of the HEMT transistor. Silvaco has models for four types of microscopic noise source: diffusion noise, Impact ionization, generationrecombination noise, and flicker noise. Diffusion noise is caused by variations in the velocity of the carrier. Generation- recombination noise is caused by variations in the number of the carriers. There are two types of generation-recombination noise in silvaco: direct and trap assisted. Direct generation-recombination noise is when the electron travels directly from the conduction band to the valence band. Trap assisted is when the electron travels from a band to a trap level. Impact ionization is very important noise in AlGaN/GaN HEMT transistor. For each electron crated in the conduction band, a corresponding hole is also created in the valence band. This is similar to direct generation-recombination noise without a recombination term. The different noise figure models introduced for heterostructure devices.Van der Ziel, Pucel, Fukui and Pospieszalski is the basis model of noise figure for HEMTs [2]. Fukui model is used in this paper for showing microwave noise performance of the proposed structure. The noise parameters of this model are [2]:

$$
\begin{aligned}
& N F_{\text {min }}=1+k_{1} \times f \times C_{g s} \times \sqrt{\frac{R s+R G}{g m}} \\
& R_{o p t}=K_{3} \times\left[\frac{1}{4 g m}+R S+R G\right] \\
& X_{o p t}=\frac{K_{4}}{f \times c g s}
\end{aligned}
$$

The variables $\mathrm{K} 1$ to $\mathrm{K} 4$, are the fitting parameters, and will change with the device technology and bias. Fig. 6 and fig. 7 shows a minimum noise figure (NFmin) and maximum associated power gain (Gma) versus frequency for the $0.25 \mathrm{um} \mathrm{AlGaN} / \mathrm{GaN} \mathrm{HEMT}$. The device was biased at $\mathrm{Vds}=10 \mathrm{~V}$ and $\mathrm{Vgs}=-3 \mathrm{~V}$. Device exhibited a NFmin of $0.35 \mathrm{~dB}$ and a (Gma) of $24.35 \mathrm{~dB}$ at $10 \mathrm{GHz}$. In the frequency limit of $3 \mathrm{GHz}-20$ $\mathrm{GHz}$ Gma ranges from $29.12 \mathrm{~dB}$ to $21.82 \mathrm{~dB}$ and NFmin is in the range of $0.14 \mathrm{~dB}$ to $0.54 \mathrm{~dB}$. Aluminum mole fraction increment plays major role in noise decrement in the proposed structure. Aluminum mole fraction increment in the $\mathrm{AlGaN}$ layer, increases the polarized carrier density and hence the electric filed is increased. Aluminum mole fraction increment in the AlGaN layer results in the 2DEG depth increment. As a result, electron density in the $2 \mathrm{DEG}$ region is increased. Therefore, device transconductance (gm) increases and due to equation (1), the device noise is reduced.

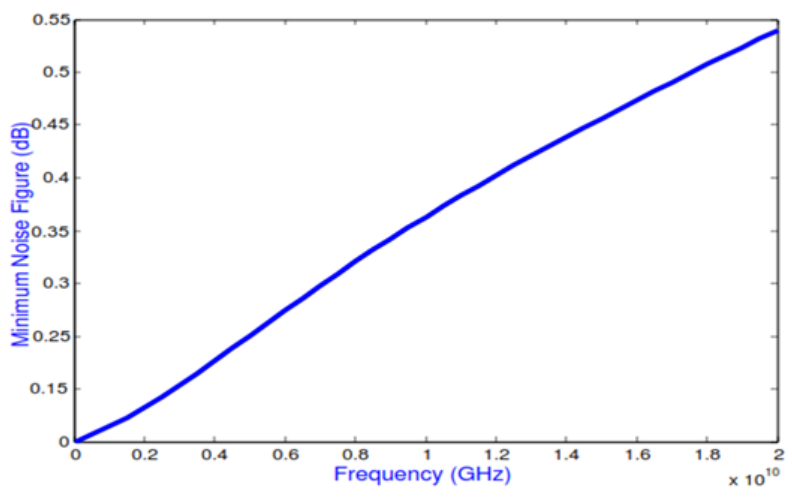

Fig. 6. $(\mathrm{NFmin}-\mathrm{F})$, at the $\mathrm{Vds}=10 \mathrm{~V}$ and $\mathrm{Vgs}=-3 \mathrm{~V}$

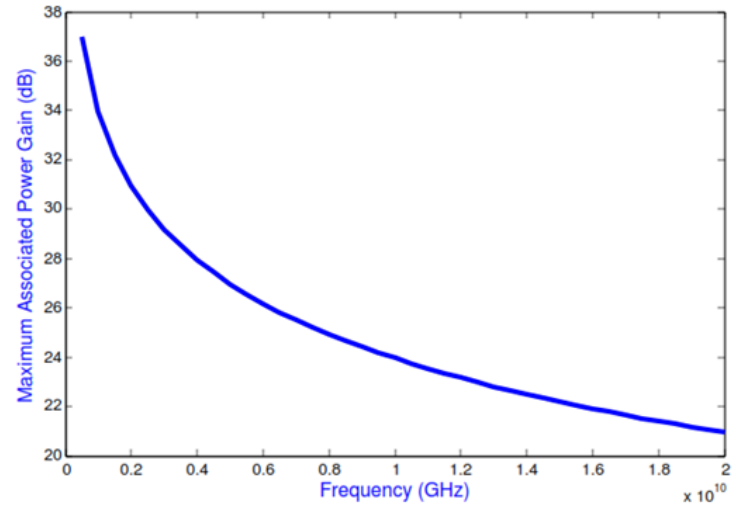

Fig. 7. (Gma-F), at the $\mathrm{Vds}=10 \mathrm{~V}$ and $\mathrm{Vgs}=-3 \mathrm{~V}$ 
Fig. 10 indicate the unity current gain cut-off frequencies $(\mathrm{Ft})$ of device against frequency at $\mathrm{Vds}=12 \mathrm{v}$ and $\mathrm{Vgs}$ $=-5.5 \mathrm{~V}$. The $(\mathrm{Ft})$ of device is equal to $200 \mathrm{GHz}$.

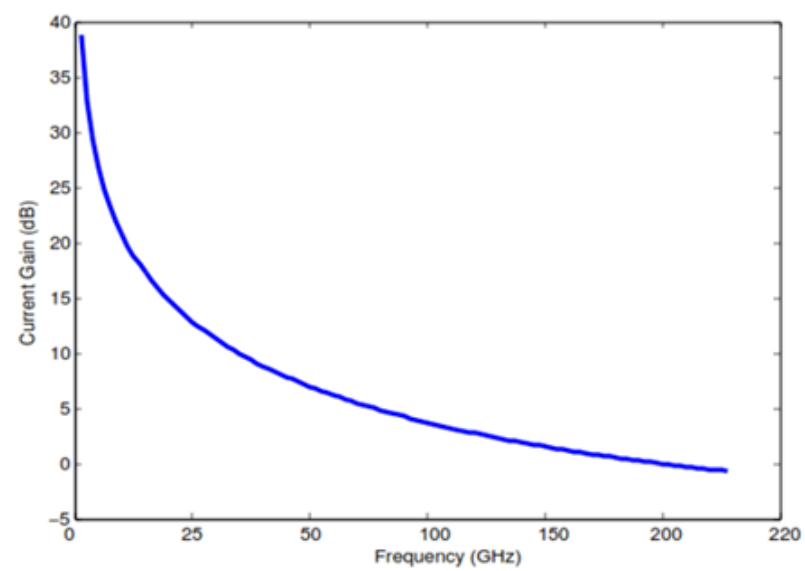

Fig. 10. Current gain cut-off frequencies $(\mathrm{Ft})$ as a function of frequency

\section{Conclusion}

In this paper, $\mathrm{AlGaN} / \mathrm{GaN} / \mathrm{AlGaN} / \mathrm{GaN}$ HEMT on Sic substrate with a gate length of $0.25 \mathrm{um}$ has been designed and simulated. The device exhibited a high current drive capability of $1750 \mathrm{~mA} / \mathrm{mm}$. The peak extrinsic transconductance of device is equal to $350 \mathrm{mS} / \mathrm{mm}$. The microwave noise performance of proposed structure was explored. The device exhibited excellent noise performance. An NFmin of $0.35 \mathrm{~dB}$ and a Gma of $24.35 \mathrm{~dB}$ have been obtained at $10 \mathrm{GHz}$ and $\mathrm{Vds}=10 \mathrm{~V}$ as follow as $\mathrm{Vgs}=-3 \mathrm{~V}$. All these superb DC and microwave noise characteristics indicate the potential of AlGaN/GaN-HEMT transistor for design of low noise amplifier in microwave frequency range. The simulation results have good assent with desired demand.

\section{References}

[1]. Wu Lu, Jinwei Yang, M. Asif Khan and Ilesanmi Adesida, AlGaN/GaN HEMTs on SiC with over $100 \mathrm{GHz} f \mathrm{f}$ and Low Microwave Noise, IEEE TRANSACTIONS ON ELECTRON DEVICES, VOL. 48, NO. 3, 2001.

[2]. Sonia sadeghi, Mehdi vadizadeh and Rahim faez, Compare Noise Characteristic of DC-HEMT and HEMT, Electrical Engineering (ICEE), 2013 21st Iranian Conference on, , 2013, 1-4.

[3]. J.-W. Lee, A. Kuliev, V. Kumar, R. Schwindt and I. Adesida, Microwave Noise Characteristics of AlGaN/GaN HEMTs on SiC Substrates for Broad-Band Low-Noise Amplifiers, IEEE MICROWAVE AND WIRELESS COMPONENTS LETTERS, VOL. 14, NO. 6, 2004.

[4]. A. T. Ping et al, Microwave noise performance of AlGaN/GaN HEMTs, Electron. Letters, vol. 36, 2000, 175-176.

[5]. N. X. Nguyen et al, Robust low microwave noise GaN MODFET's with $0.60 \mathrm{~dB}$ noise figure at $10 \mathrm{GHz}$, Electron. Letters, vol. 36, 2000, 469-471.

[6]. I. P. Smorchkova, M. Wojtowicz, R. Sandhu, R. Tsai, M. Barsky, C. Namba, P.-S. Liu, R. Dia, M. Truong, D. Ko, J. Wang, H. Wang, and A. Khan, AlGaN/GaN HEMTs-operation in the K-band and above, IEEE TRANSACTIONS Microwave Theory Tech, vol. 51, 2003, 665-668

[7]. X. D. Wang, W. D. Hu, X. S. Chen and Wei lu, the study of self heating and hot electron effects for AlGaN/GaN Double -Channel HEMTs, IEEE TRANSCTION ON ELECTRON DEVICE,vol.59,no.5, 2012.

Alireza Kashaniniya has received his B.Sc in electronics communication engineering (1995), M.Sc (1998) and

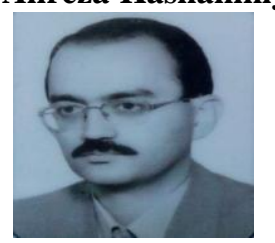
Ph.D (2005) in electronics engineering from University of Tehran and Science and Researches Univ., Tehran, Iran. He is now assistant professor in IAUCTB Tehran, Iran. The research interests of Dr. Kashaniniya includes high frequencies and nano scale transistors, RFICs, micro and nano scale devices and sensors, implanable circuits and devices for biomedical applications.

Yasaman Najmabadi was born in Tehran, Iran, in 1991. She received the B.Sc. Degree in electrical

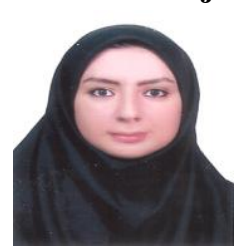
engineering (electronic) from the North Branch, Islamic Azad University, Tehran, Iran in 2013. She is first ranked among all B.Sc. Students in the department of electrical engineering. I am a M.Sc. Student in Central Tehran Branch, Islamic Azad University, Tehran, Iran, Where she is currently working in the electronic lab. Her research interests include Design and Simulation of Semiconductor Devices, High Frequency Circuits Design (Low Noise Amplifier, High Power Amplifier) 